\title{
LA-UR-15-29547
}

Approved for public release; distribution is unlimited.

Title: $\quad$ A More General Solution of the Kenamond HE Problem 2

Author(s): Kaul, Ann

Intended for: Report

Issued: 
Disclaimer:

Los Alamos National Laboratory, an affirmative action/equal opportunity employer,is operated by the Los Alamos National Security, LLC for the National NuclearSecurity Administration of the U.S. Department of Energy under contract DE-AC52-06NA25396. By approving this article, the publisher recognizes that the U.S. Government retains nonexclusive, royalty-free license to publish or reproduce the published form of this contribution, or to allow others to do so, for U.S. Government purposes. Los Alamos National Laboratory requests that the publisher identify this article as work performed under the auspices of the U.S. Departmentof Energy. Los Alamos National Laboratory strongly supports academic freedom and a researcher's right to publish; as an institution, however, the Laboratory does not endorse the viewpoint of a publication or guarantee its technical correctness. 


\section{A More General Solution of the Kenamond HE Problem 2}

The Kenamond High Explosives (HE) Problem Set is a series of problems designed to test the burn table solution of HE lighting times generated for programmed burn simulations. The suite of test problems has exact solutions in 2D and 3D. The suite of problems was originally defined in Reference 1 and further studied in Reference 2.

It should be understood that these burn time calculations are purely geometry-based solutions. They do not account for HE behaviors such as shock formation time, inert boundary behaviors or behavior at boundaries between two HEs.

\section{Problem Description}

The Kenamond HE Problem 2 is used to test a code's ability to calculate burn tables for an unobstructed line-of-sight, multi-point initiation of a composite HE region. The problem is described as follows: An HE sphere of radius $R$ centered at the origin with constant detonation velocity $D_{1}$ is surrounded by an infinite medium of a second HE with constant detonation velocity $D_{2}$. Five point detonators located at $\boldsymbol{x}=\boldsymbol{x}_{d, i}$ are ignited at times $t=t_{d, i}$. Figure 1 shows a specific case of this problem where the detonators are located on the $y$-axis in the $2 \mathrm{D}$ problem or on the $z$-axis in the 3D problem. This is the problem described in References 1 and 2.

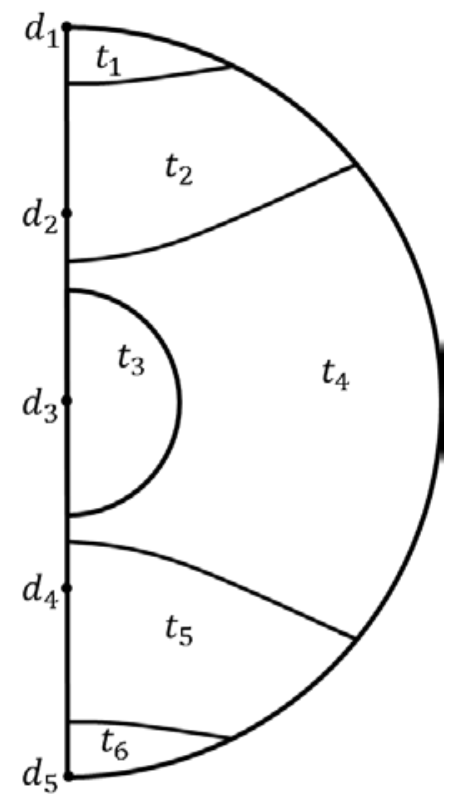

Figure 1. Initial configuration of a specific case of the Kenamond HE Problem 2. The regions are labeled by the particular burn time solution which applies in that region.

The given solutions were developed with the center of the inert region located at the origin. This is not strictly necessary and the center could be located at any point. In that case, all vector quantities in the solutions given in the following sections would need to be translated by the vector from the origin to the specified center. This generality does not produce any meaningful 
contribution to the calculation, however. Therefore, the origin was chosen as the center of the inner HE region, as that provides for some degree of simplification of the solution expressions.

The HE burn time solution is independent of the initial state parameters (such as density or temperature) specified for the HE materials. It depends only on the size of the inert object and on the specified detonation times, detonator positions and HE detonation velocities.

\section{General Solution}

One physical consideration for this problem is the relative detonation velocities of the two HE materials. It is difficult to predict the behavior of an explosive's detonation front when it interacts with a HE with a higher detonation velocity. Thus, the most general solution proposed in this document will assume that the detonation velocity of the inner region is higher than that of the outer region:

$$
D_{1}>D_{2}
$$

As a result, the entire inner $\mathrm{HE}$ region will need to be ignited by a detonator located inside that region.

To that end, detonator 3 will always be located at the center of the inner HE region (the origin) so that its detonation front will exit the inner region simultaneously at all points on the interface between HE regions. The other four detonators are placed at points in the outer HE region such that their detonation fronts do not reach the surface of the inner region before the wave from detonator 3 exits that region. This places a time / position constraint on the other detonators. To find it, compare arrival times at the surface of the inner region. The burn front from detonator 3 will arrive everywhere at the surface of the inner region, which is located at $\|x\|=R$, at time

$$
t_{R, 3}=t_{d, 3}+\frac{R}{D_{1}}
$$

Meanwhile, the burn front from any other detonator will reach the surface of the inner region at time

$$
t_{R, i}=t_{d, i}+\frac{\left\|x_{d, i}\right\|-R}{D_{1}}
$$

Since the detonator 3 burn front must arrive first, $t_{R, i} \geq t_{R, 3}$, which gives the following condition on the position and detonation time of the other four detonators:

$$
t_{d, i} \geq t_{d, 3}+R\left(\frac{1}{D_{1}}+\frac{1}{D_{2}}\right)-\frac{\left\|x_{d, i}\right\|}{D_{2}}
$$

For the most general case as described above, with detonator 3 located at the origin, the burn time solution at the point located at $\boldsymbol{x}=\boldsymbol{p}$ is as follows:

$$
t(\boldsymbol{x})=\min \left(t_{1}, t_{2}, \max \left(t_{3}, t_{4}\right), t_{5}, t_{6}\right)
$$

where

$$
t_{1}(\boldsymbol{x})=t_{d, 1}+\frac{\left\|\boldsymbol{x}-\boldsymbol{x}_{d, 1}\right\|}{D_{2}}
$$




$$
\begin{gathered}
t_{2}(\boldsymbol{x})=t_{d, 2}+\frac{\left\|\boldsymbol{x}-\boldsymbol{x}_{d, 2}\right\|}{D_{2}} \\
t_{3}(\boldsymbol{x})=t_{d, 3}+\frac{\|\boldsymbol{x}\|}{D_{1}} \\
t_{4}(\boldsymbol{x})=t_{d, 3}+\frac{\|\boldsymbol{x}\|}{D_{2}}+R\left(\frac{1}{D_{1}}-\frac{1}{D_{2}}\right) \\
t_{5}(\boldsymbol{x})=t_{d, 4}+\frac{\left\|\boldsymbol{x}-\boldsymbol{x}_{d, 4}\right\|}{D_{2}} \\
t_{6}(\boldsymbol{x})=t_{d, 5}+\frac{\left\|\boldsymbol{x}-\boldsymbol{x}_{d, 5}\right\|}{D_{2}}
\end{gathered}
$$

It should be noted that the general solution given above does not depend on the dimensionality of the problem and holds for both 2D and 3D geometries.

In addition to determining the burn time at a particular point, it is useful to know which detonator is responsible for igniting that point. The interfaces between detonation fronts will define this answer. To find the interfaces, consider that the detonator time/position condition about prevents any other detonation wave from burning the inner region. In addition, at the surface of the inner region $(\|x\|=R)$, it can be shown that $t_{3}(\boldsymbol{x})=t_{4}(\boldsymbol{x})$. Thus, the region defined by $t_{3}(\boldsymbol{x})$ does not intersect the regions defined by $t_{i}(\boldsymbol{x}), i=1,2,5,6$ except perhaps at isolated points. That leaves only two cases to consider: either these regions intersect the region defined by $t_{4}(\boldsymbol{x})$ or they intersect each other.

In the first case, a burn front from detonator 1, 2, 4 or 5 meets the burn front from detonator 3, with the intersection defined by

$$
t_{4}(\boldsymbol{x})=t_{i}(\boldsymbol{x}) ; \quad i=1,2,5,6
$$

Substituting the above expressions and rearranging them gives

$$
\left\|x-x_{d, i}\right\|-\|x\|=D_{2}\left(t_{d, 3}-t_{d, i}\right)+R\left(\frac{D_{2}}{D_{1}}-1\right)
$$

All of the quantities on the right-hand side are constants for a given problem. Thus, the intersection of the burn fronts from detonators 3 and one of the other detonators is one branch of a hyperbola in 2D and of a hyperboloid in 3D.

In the second case, a burn front from detonator 1, 2, 4 or 5 meets the burn front from another of these detonators. Here, the intersection is defined by

Substituting and rearranging gives

$$
t_{i}(\boldsymbol{x})=t_{j}(\boldsymbol{x}) ; i, j=1,2,5,6 ; i \neq j
$$

$$
\left\|\boldsymbol{x}-\boldsymbol{x}_{d, i}\right\|-\left\|\boldsymbol{x}-\boldsymbol{x}_{d, j}\right\|=D_{2}\left(t_{d, j}-t_{d, i}\right)
$$

Again, all of the quantities on the right-hand side are constants for a given problem. The intersection of the burn fronts from any two of the other detonators is also one branch of a hyperbola in 2D and of a hyperboloid in 3D. 


\section{Solutions for Axial Detonators}

The verification test problems implemented in the Verification Test Suite (VTS) use the problem's inherent symmetry to reduce the computational load. In 2D, a typical simulation will calculate the burn times for a semi-circle, with the detonators located on the symmetry axis. In 3D, a typical simulation will calculate the burn times for a spherical wedge, with the detonators again located on the symmetry axis. The simplest symmetry for code simulations places the detonators on a Cartesian axis.

In a 2D geometry, the detonators can be located on either the $x$-axis or $y$-axis. In 3D geometry, the detonators are typically located on the $z$-axis. The Kenamond2 solver of the ExactPack verification solver suite implements the solution for detonators located on the $y$-axis in 2D, with $\boldsymbol{x}_{d, i}=\left\langle 0, y_{d, i}\right\rangle$, and on the $z$-axis in 3D, with $\boldsymbol{x}_{d, i}=\left\langle 0,0, z_{d, i}\right\rangle$. This allows the detonator positions to be specified with one number per detonator.

While it is straight-forward to rewrite the burn time solution equations from the previous section using components in place of the vectors, this change will not provide any new information. It is worth noting that the detonation time / detonator position constraint outlined above will become in the $2 \mathrm{D}$ case

In the 3D case, the constraint becomes

$$
t_{d, i} \geq t_{d, 3}+R\left(\frac{1}{D_{1}}+\frac{1}{D_{2}}\right)-\frac{\left|y_{d, i}\right|}{D_{2}}
$$

$$
t_{d, i} \geq t_{d, 3}+R\left(\frac{1}{D_{1}}+\frac{1}{D_{2}}\right)-\frac{\left|z_{d, i}\right|}{D_{2}}
$$

In addition, it can be shown that the 2D hyperbolic intersections of the burn fronts are centered on the $y$-axis, while the 3D hyperboloid intersections are centered on the $z$-axis.

The solution implemented in the ExactPack suite includes the position / time condition for the axially-located detonators. The more general case could be implemented in ExactPack with two changes. The detonator position inputs would need to become 2D or 3D tuples. The detonator time / position constraint would need to be changed to the general location constraint from the previous section.

\section{Comparison to the Original Solutions}

In the original paper (Reference 1), a solution was developed for a series of detonators as shown in Figure 1, above. The specific detonator positions and detonation times are listed in Table 1.

\begin{tabular}{|c|c|c|c|}
\hline Detonator $(i)$ & $t_{d, i}$ & $\boldsymbol{x}_{d, i}$ (2D Cartesian) & $\boldsymbol{x}_{d, i}$ (3D Cartesian) \\
\hline 1 & 2.0 & $(0.0,10.0)$ & $(0.0,0.0,10.0)$ \\
\hline 2 & 1.0 & $(0.0,5.0)$ & $(0.0,0.0,5.0)$ \\
\hline 3 & 0.0 & $(0.0,0.0)$ & $(0.0,0.0,0.0)$ \\
\hline 4 & 1.0 & $(0.0,-5.0)$ & $(0.0,0.0,-5.0)$ \\
\hline 5 & 2.0 & $(0.0,-10.0)$ & $(0.0,0.0,-10.0)$ \\
\hline
\end{tabular}

Table 1. Detonator locations and detonation times for the original Kenamond HE Problem 2. 
The radius of the inner HE region was specified as $R=3.0$. The detonation velocities were given as $D_{1}=2.0$ and $D_{2}=1.0$. It can easily be shown that the detonators specified in Table 1 satisfy the time / position constraint given above.

The burn time solution then becomes

$$
t(\boldsymbol{x})=\min \left(t_{1}, t_{2}, \max \left(t_{3}, t_{4}\right), t_{5}, t_{6}\right)
$$

where

$$
\begin{gathered}
t_{1}(\boldsymbol{x})=\left\|\boldsymbol{x}-\boldsymbol{x}_{d, 1}\right\|+2 \\
t_{2}(\boldsymbol{x})=\left\|\boldsymbol{x}-\boldsymbol{x}_{d, 2}\right\|+1 \\
t_{3}(\boldsymbol{x})=\|\boldsymbol{x}\| / 2 \\
t_{4}(\boldsymbol{x})=\|\boldsymbol{x}\|-1.5 \\
t_{5}(\boldsymbol{x})=\left\|\boldsymbol{x}-\boldsymbol{x}_{d, 4}\right\|+1 \\
t_{6}(\boldsymbol{x})=\left\|\boldsymbol{x}-\boldsymbol{x}_{d, 5}\right\|+2
\end{gathered}
$$

The interfaces between the burn fronts will be defined first for the 2D case. The burn fronts from detonators 1 and 2 will intersect on the hyperbola defined by $t_{1}(\boldsymbol{x})$ and $t_{2}(\boldsymbol{x})$ :

$$
24\left(y-\frac{15}{2}\right)^{2}-x^{2}=6
$$

Since the two burn fronts must meet at $(0,8)$ on the $y$-axis, the solution branch is given by

$$
y=\frac{15}{2}+\sqrt{\frac{1}{4}+\frac{x^{2}}{24}}
$$

The burn fronts from detonators 2 and 3 will intersect on the hyperbola defined by $t_{2}(\boldsymbol{x})$ and $t_{4}(\boldsymbol{x})$ :

$$
3\left(y-\frac{5}{2}\right)^{2}-x^{2}=\frac{75}{16}
$$

The two burn fronts must meet at $(0,3.75)$ on the $y$-axis, so the solution branch is given by

$$
y=\frac{5}{2}+\sqrt{\frac{25}{16}+\frac{x^{2}}{3}}
$$

The burn fronts from detonators 3 and 4 will intersect on the hyperbola defined by $t_{4}(\boldsymbol{x})$ and $t_{5}(\boldsymbol{x})$ :

$$
3\left(y+\frac{5}{2}\right)^{2}-x^{2}=\frac{75}{16}
$$

These two burn fronts must meet at $(0,-3.75)$ on the $y$-axis; thus, the solution branch is given by

$$
y=-\frac{5}{2}-\sqrt{\frac{25}{16}+\frac{x^{2}}{3}}
$$

For detonators 1 and 2, the burn fronts will intersect on the hyperbola defined by $t_{5}(\boldsymbol{x})$ and $t_{6}(\boldsymbol{x})$ : 


$$
24\left(y+\frac{15}{2}\right)^{2}-x^{2}=6
$$

Because the two burn fronts must meet at $(0,-8)$ on the $y$-axis, the solution branch is given by

$$
y=-\frac{15}{2}-\sqrt{\frac{1}{4}+\frac{x^{2}}{24}}
$$

The regions in Figure 1 are labeled by the time solution valid in that region.

The solution for the 3D geometry, which assumes that the detonators are located on the $z$-axis with $\boldsymbol{x}_{d, i}=\left\langle 0,0, z_{d, i}\right\rangle$, can now be easily found by considering the diagram in Figure 1 to be in cylindrical coordinates. In other words, let the vertical $y$-axis become the $z$-axis and the horizontal $x$-axis become the $r$-axis. Then, the previous solution can be modified to find the interfaces of the burn fronts in 3D by replacing $y$ with $z$ and $x$ with $r=\sqrt{x^{2}+y^{2}}$.

\section{Conclusion}

A more general solution for programmed burn calculations of the light times produced by an unobstructed line-of-sight, multi-point initiation of a composite HE region has been developed. The equations describing the interfaces between detonation fronts have also been included. In contrast to the original solutions proposed in References 1 and 2, four of the detonators are no longer restricted to specific locations on a Cartesian axis and can be located at any point inside the HE region. For the proposed solution, one detonator must be located at the origin. The more general solution for any locations on the 2D $y$-axis or 3D $z$-axis has been implemented in the ExactPack suite of exact solvers for verification problems. It could easily be changed to the most general case outlined above.

\section{References}

1. Kenamond, M. A., "Line-of-Sight High Explosive Programmed Burn Model in the FLAG Code”, LA-UR 09-08296, 2009.

2. Kenamond, M. A., “HE Burn Table Verification Problems”, LA-UR 11-03096, 2011. 